\title{
Efficacy of Insecticides and Bio Pesticides against Sucking Insect Pests on Bt Cotton
}

\author{
H. Meghana*, S. B. Jagginavar and N. D. Sunitha \\ Department of Agricultural Entomology, College of Agriculture, Vijayapur. University of \\ Agricultural Sciences, Dharwad-580005, Karnataka, India \\ *Corresponding author
}

\section{A B S T R A C T}

\section{Keywords \\ Bt cotton, thrips, aphids, jassids, whitefly, insecticides and bio pesticides \\ Article Info \\ Accepted: \\ 20 May 2018 \\ Available Online: \\ 10 June 2018}

A field experiment was conducted to evaluate the efficacy of insecticides and bio pesticides against sucking insect pests on Bt cotton at College of Agriculture, Vijayapur during kharif-2016-17. The results revealed that the treatment flonicamid 50 WG was observed as significantly superior insecticide in minimizing the thrips population followed by fipronil $5 \mathrm{SC}$, acetamiprid $20 \mathrm{SP}$, dinotefuron $20 \mathrm{SG}$, diafenthiuron $50 \mathrm{WP}$ and thiamethoxam 25 WG. However, significantly least population of aphids were observed from the plots which received flonicamid $50 \mathrm{WG}$, acetamiprid $20 \mathrm{SP}$, dinotefuran $20 \mathrm{SG}$ and fipronil $5 \mathrm{SC}$, thiamethoxam $25 \mathrm{WG}$ and diafenthiuron $50 \mathrm{WP}$. With respect to jassids and whiteflies, the least population were recorded from the plot treated with flonicamid 50 WG, dinotefuran $20 \mathrm{SG}$, diafenthiuron $50 \mathrm{WP}$, acetamiprid $20 \mathrm{SP}$, thiamethoxam $25 \mathrm{WG}$ and fipronil 5 SC compared to untreated control.

\section{Introduction}

Cotton (Gossypium spp.) is one of the most commercially important fiber crops in the world. It is an important raw material for the Indian textile industry and plays a key role in the national economy in terms of both employment generation and foreign exchange. Due to its economic importance it is commonly called as "white gold". Which is being cultivated in an area of about 11.8 million ha with 27.8 million bales of production and $513 \mathrm{~kg} / \mathrm{ha}$ of average productivity in India. Cotton is known to attack by a wide range of insect pests at various stages of growth (Uthamasamy, 1994). Now a days sucking pests are causing severe threat in cotton ecosystem. However, the major sucking pests viz., jassids, thrips, aphids, whitefly and mirid bugs etc are continuously damaging and causing considerable yield loss in Bt cotton. Thus, keeping these points in view, the present study was undertaken to determine the field efficacy of different insecticides and bio pesticides against sucking insect pests

\section{Materials and Methods}

An investigation was conducted at College of Agriculture, Vijayapur, to study the efficacy of insecticides and bio pesticides against sucking insect pests on Bt cotton under rainfed situation during kharif season 2016-17 in a 
randomized block design (RBD) with 10 treatments and were replicated thrice. The popular Bt cotton hybrid of Vijayapur district, ACH-155 BG II was selected for the investigation with spacing of $90 \times 60 \mathrm{~cm}$ in the plot size of $4.2 \times 6.3 \mathrm{~m}^{2}$. The crop was raised as per the package of practices (Anon., 2014) except for plant protection measures.

\section{Treatment details}

T1: Diafenthiuron $50 \mathrm{WP}(0.6 \mathrm{~g} / \mathrm{l})$

T2: Dinotefuran 20 SG (0.3g/l)

T3: Fipronil 5 SC (1 ml/l)

T4: Flonicamid $50 \mathrm{WG}(0.3 \mathrm{~g} / \mathrm{l})$

T5: Quinalphos 25 EC (2ml/l)

T6: Thiamethoxam 25 WG $(0.2 \mathrm{~g} / \mathrm{l})$

T7: Nimbecidine 1500 ppm (2 ml /l)

T8: Lecanicillium lecanii $2 \times 10^{-8} \mathrm{cfu} / \mathrm{g}(5 \mathrm{~g} / \mathrm{l})$

T9: Acetamiprid 20 SP (Standard check) $(0.2 \mathrm{~g} / \mathrm{l})$

T10: Untreated control

The observations on sucking pests like thrips, aphids, jassids and whiteflies were recorded as number of nymphs and adults per top, middle and bottom 3 leaves on randomly selected 5 plants from each treatment, later the population was averaged as number per 3 leaves. All the insecticidal treatments were imposed four times according to the crop growth stages at 40, 55, 70 and 90 days after sowing. Observation was recorded at one day before, 3, 5 and 10 days after each spray. Care was taken to avoid the drifting of spray droplets between the treatments by placing polythene sheet as physical barrier.

\section{Statistical analysis}

The data obtained were subjected to square root transformation $(\sqrt{ } \mathrm{x}+0.5)$ and were analyzed by following analysis of variance and treatment means were compared by following Duncan's Multiple Range Test (DMRT) as suggested by Gomez and Gomez (1984).

\section{Results and Discussion}

\section{Thrips}

The data regarding mean population of thrips/ 3 leaves at first, second, third and fourth spray were presented in Table 1 and 2. The result revealed that at three days after first spray, significantly lowest thrips population of 35.36 thrips / 3 leaves was recorded from flonicamid $50 \mathrm{WG}$ treated plot followed by fipronil $5 \mathrm{SC}$ (37.25 thrips / 3 leaves), acetamiprid 20 SP (41.29 thrips / 3 leaves) and dinotefuron 20 SG (41.74 thrips / 3 leaves). These treatments were significantly superior and on par with each other. The treatments diafenthiuron 50 WP (45.50 thrips / 3 leaves) and thiamethoxam $25 \mathrm{WG}$ (45.94 thrips / 3 leaves) were emerged as next best than the rest of the treatments. During five, ten days after first spray, second, third and fourth spray also similar trend was noticed with regard to reduction in thrips population. Present investigation clearly indicates that insecticidal treatments were highly efficient in managing the sucking insect pests than bio pesticides. Present study results are in close agreement with findings of Bhavani Sankara Rao et al., (1991) and Ghelani et al., (2006) they also reported that bio pesticides (botanicals and microbials) were less effective over the chemical pesticides against sucking pests of okra. Gaurkhede et al., (2015) reported that fipronil $5 \mathrm{SC}$, flonicamid $50 \mathrm{WG}$, dinotefuran 20 SG and acetamiprid 20 SP effectively minimized the thrips density. Ghelani et al., 
(2014) and Ravikumar et al., (2016) observed maximum mortality of thrips with flonicamid 50 WG. Similar findings were also documented by Sathyan et al., (2016) and Patil et al., (2009). The present findings are contradictory with the findings of Bharpoda et al., (2014) as they reported that fipronil 5 EC and thiamethoxam $25 \mathrm{WG}$ were less effective and recorded higher thrips population.

\section{Aphids}

The aphid incidence was not noticed during $1^{\text {st }}$ and $2^{\text {nd }}$ sprays. The population was started to appear in the month of September, hence only $3^{\text {rd }}$ and $4^{\text {th }}$ sprays observation was taken. In the present investigation the treatments flonicamid $50 \mathrm{WG}$, acetamiprid $20 \mathrm{SP}$, dinotefuran $20 \mathrm{SG}$ and fipronil $5 \mathrm{SC}$ were significantly superior and equally effective against aphids over rest of the treatments $(7.33,7.83,7.91 \& 8.87$ aphids / three leaves, respectively) at 3 days after $3^{\text {rd }}$ spray and were on par with each other. The next best treatments were thiamethoxam $25 \mathrm{WG}$ (9.07 aphids / 3 leaves), diafenthiuron 50 WP (9.26 aphids / 3 leaves) and quinalphos $25 \mathrm{EC}$ (11.17 aphids / 3 leaves) these were significantly superior to flonicamid 50 WG (7.33 aphids / 3 leaves) (Table 3). Similarly, during five, ten days after third spray and fourth spray same trend of superiority was observed.

The present investigations are in line with the outcome of Ghelani et al., (2014) they also reported that the treatments with flonicamid, acetamiprid, thiamethoxam caused significantly maximum mortality of aphids. Gaurkhede et al., (2015) observed minimum aphid population in the plots treated with flonicamid 50 WG @ 0.02 per cent (2.96 aphids/ leaf) and was at par with dinotefuran 20 SG @ 0.008 (3.50 aphids / leaf). Sathyan et al., (2016) reported that quinalphos $25 \mathrm{EC}$ and flubendiamide $20 \mathrm{WG}$ were found to be less effective against aphid population.

\section{Jassids}

Minimum population of jassids was observed in flonicamid $50 \mathrm{WG}(0.18$ jassids / 3 leaves $)$ and dinotefuran $20 \mathrm{SG}$ (0.21 jassids / 3 leaves), which were equally superior and on par with one another.

The next superior treatments with minimum incidence of jassids were diafenthiuron 50 WP, acetamiprid $20 \mathrm{SP}$, thiamethoxam $25 \mathrm{WG}$ and fipronil $5 \mathrm{SC}$ with the mean population of $0.26,0.29,0.33$ and 0.34 jassids / 3 leaves, respectively these were statistically at par with each other (Table 4 and 5). Similar trend was noticed in all the spray with regard to reduction in jassid population. Similar results were obtained by Kadam et al., (2014) they also revealed that maximum mortality of jassid was found in flonicamid treated plot.

Razaq et al., (2005) they also found minimum population of jassids ( 0.43 jassid / leaf) in plots treated with acetamiprid followed by thiamethoxam (0.95 jassid / leaf), diafenthiuron (1.06 jassid / leaf) at seven days after application. Gaurkhede et al., (2015) reported dinotefuran 20 SG @ 0.008 per cent (0.91 jassids/ leaf), dinotefuran 20 SG @ 0.006 per cent (1.01 jassids / 3 leaves) fipronil 5 SC @ 0.015 per cent (1.12 jassids / 3 leaves), acetamiprid 20 SP @ 0.004 per cent (1.16 jassids / 3 leaves), imidacloprid 30.5 SC @ 0.005 per cent ( 1.21 jassids / 3 leaves) and flonicamid 50 WG @ 0.02 per cent (1.28 jassids / 3 leaves) did not differ significantly in minimizing the jassids population.

\section{Whitefly}

The treatments flonicamid 50 WG, dinotefuran $20 \mathrm{SG}$ and diafenthiuron $50 \mathrm{WP}$ were significantly superior over other insecticides with regard to whitefly suppression and the whitefly incidence was nil in all these three treatments at 3 DAS. 
Table.1 Efficacy of insecticides and bio pesticides against thrips, Thrips tabaci (Lindeman) on Bt cotton ( $1^{\text {st }}$ and $2^{\text {nd }}$ spray)

\begin{tabular}{|c|c|c|c|c|c|c|c|c|c|}
\hline \multirow{3}{*}{$\begin{array}{c}\text { S. } \\
\text { No }\end{array}$} & \multirow[t]{3}{*}{ Treatments } & \multicolumn{8}{|c|}{ Mean population of thrips / 3 leaves } \\
\hline & & \multicolumn{4}{|c|}{ First spray } & \multicolumn{4}{|c|}{ Second spray } \\
\hline & & 1 DBS & 3 DAS & 5 DAS & 10 DAS & $1 \mathrm{DBS}$ & 3 DAS & 5 DAS & 10 DAS \\
\hline 1. & Diafenthiuron 50 WP @ 0.6g/ litre & $\begin{array}{l}74.49 \\
(8.66)\end{array}$ & $\begin{array}{c}45.50 \\
(6.78)^{\mathrm{ab}}\end{array}$ & $\begin{array}{c}41.18 \\
(6.46)^{\mathrm{ab}}\end{array}$ & $\begin{array}{c}39.45 \\
(6.32)^{\mathrm{abcd}}\end{array}$ & $\begin{array}{c}47.19 \\
(6.91)^{\mathrm{bc}}\end{array}$ & $\begin{array}{c}28.47 \\
(5.38)^{\mathrm{bc}}\end{array}$ & $\begin{array}{c}22.57 \\
(4.80)^{\mathrm{bc}}\end{array}$ & $\begin{array}{c}6.13 \\
(2.56)\end{array}$ \\
\hline 2. & Dinotefuran 20 SG @ 0.3g/litre & $\begin{array}{l}72.71 \\
(8.56)\end{array}$ & $\begin{array}{c}41.74 \\
(6.49)^{\mathrm{a}}\end{array}$ & $\begin{array}{c}37.52 \\
(6.16)^{\mathrm{ab}}\end{array}$ & $\begin{array}{c}36.09 \\
(6.05)^{\mathrm{abc}}\end{array}$ & $\begin{array}{c}43.64 \\
(6.64)^{\mathrm{abc}}\end{array}$ & $\begin{array}{c}21.79 \\
(4.72)^{\mathrm{ab}}\end{array}$ & $\begin{array}{c}16.69 \\
(4.14)^{\mathrm{ab}}\end{array}$ & $\begin{array}{c}5.45 \\
(2.43)\end{array}$ \\
\hline 3. & Fipronil 5 SC @1 ml/litre & $\begin{array}{l}75.48 \\
(8.71)\end{array}$ & $\begin{array}{l}37.25 \\
(6.14)^{\mathrm{a}}\end{array}$ & $\begin{array}{c}31.88 \\
(5.69)^{\mathrm{a}}\end{array}$ & $\begin{array}{c}29.83 \\
(5.50)^{\mathrm{ab}}\end{array}$ & $\begin{array}{c}36.15 \\
(6.05)^{\mathrm{ab}}\end{array}$ & $\begin{array}{c}14.64 \\
(3.89)^{\mathrm{a}}\end{array}$ & $\begin{array}{c}11.28 \\
(3.43)^{\mathrm{a}}\end{array}$ & $\begin{array}{c}4.84 \\
(2.29)\end{array}$ \\
\hline 4. & Flonicamid 50 WG @ 0.3g/litre & $\begin{array}{l}75.85 \\
(8.73)\end{array}$ & $\begin{array}{c}35.36 \\
(5.98)^{\mathrm{a}}\end{array}$ & $\begin{array}{l}28.56 \\
(5.39)^{\mathrm{a}}\end{array}$ & $\begin{array}{c}25.52 \\
(5.09)^{\mathrm{a}}\end{array}$ & $\begin{array}{c}31.80 \\
(5.68)^{\mathrm{a}}\end{array}$ & $\begin{array}{c}12.41 \\
(3.59)^{\mathrm{a}}\end{array}$ & $\begin{array}{c}9.84 \\
(3.22)^{\mathrm{a}}\end{array}$ & $\begin{array}{c}5.80 \\
(2.50)\end{array}$ \\
\hline 5. & Quinalphos 25 EC @ 2 ml/litre & $\begin{array}{l}72.45 \\
(8.54)\end{array}$ & $\begin{array}{c}55.49 \\
(7.48)^{\text {abc }}\end{array}$ & $\begin{array}{c}49.64 \\
(7.07)^{\mathrm{bc}}\end{array}$ & $\begin{array}{c}47.40 \\
(6.92)^{\mathrm{bcd}}\end{array}$ & $\begin{array}{c}59.72 \\
(7.76)^{\mathrm{cd}}\end{array}$ & $\begin{array}{c}39.42 \\
(6.32)^{\mathrm{cd}}\end{array}$ & $\begin{array}{c}32.30 \\
(5.73)^{\mathrm{cd}}\end{array}$ & $\begin{array}{c}4.59 \\
(2.25)\end{array}$ \\
\hline 6. & $\begin{array}{l}\begin{array}{l}\text { Thiamethoxam } \\
\text { g/litre }\end{array} \\
\text { 25 }\end{array}$ & $\begin{array}{l}74.01 \\
(8.63)\end{array}$ & $\begin{array}{c}45.94 \\
(6.81)^{\mathrm{ab}}\end{array}$ & $\begin{array}{l}42.05 \\
(6.52)^{\mathrm{ab}}\end{array}$ & $\begin{array}{c}40.97 \\
(6.44)^{\mathrm{abcd}}\end{array}$ & $\begin{array}{l}47.45 \\
(6.91)^{\mathrm{bc}}\end{array}$ & $\begin{array}{l}29.27 \\
(5.46)^{\mathrm{bc}}\end{array}$ & $\begin{array}{c}24.00 \\
(4.95)^{\mathrm{bc}}\end{array}$ & $\begin{array}{c}4.60 \\
(2.21)\end{array}$ \\
\hline 7. & Nimbicidin 1500 ppm @ 2 ml/litre & $\begin{array}{l}73.25 \\
(8.59)\end{array}$ & $\begin{array}{l}66.26 \\
(8.17)^{\mathrm{bc}}\end{array}$ & $\begin{array}{l}63.78 \\
(8.01)^{\mathrm{c}}\end{array}$ & $\begin{array}{l}63.14 \\
(7.98)^{\mathrm{de}}\end{array}$ & $\begin{array}{l}73.39 \\
(8.59)^{\mathrm{d}}\end{array}$ & $\begin{array}{l}61.45 \\
(7.87)^{\mathrm{e}}\end{array}$ & $\begin{array}{l}57.45 \\
(7.61)^{\mathrm{e}}\end{array}$ & $\begin{array}{l}6.65 \\
(2.67)\end{array}$ \\
\hline 8. & $\begin{array}{l}\text { Lecanicillium lecanii }\left(2 \times 10^{-8} \mathrm{cfu}\right) @ \\
5 \mathrm{~g} / \text { litre }\end{array}$ & $\begin{array}{l}73.72 \\
(8.61)\end{array}$ & $\begin{array}{c}65.75 \\
(8.13)^{\mathrm{bc}}\end{array}$ & $\begin{array}{l}60.52 \\
(7.80)^{\mathrm{c}}\end{array}$ & $\begin{array}{c}59.17 \\
(7.72)^{\text {cde }}\end{array}$ & $\begin{array}{l}71.57 \\
(8.49)^{\mathrm{d}}\end{array}$ & $\begin{array}{c}50.80 \\
(7.16)^{\mathrm{de}}\end{array}$ & $\begin{array}{l}45.05 \\
(6.74)^{\mathrm{de}}\end{array}$ & $\begin{array}{c}4.60 \\
(2.23)\end{array}$ \\
\hline 9. & $\begin{array}{l}\text { Acetamiprid } 20 \text { SP @ 0.2g/litre } \\
\text { (Std check) }\end{array}$ & $\begin{array}{l}70.03 \\
(8.39)\end{array}$ & $\begin{array}{l}41.29 \\
(6.46)^{\mathrm{a}}\end{array}$ & $\begin{array}{c}37.47 \\
(6.16)^{\mathrm{ab}}\end{array}$ & $\begin{array}{c}35.69 \\
(6.01)^{\mathrm{ab}}\end{array}$ & $\begin{array}{c}42.40 \\
(6.54)^{\mathrm{abc}}\end{array}$ & $\begin{array}{c}20.56 \\
(4.59)^{\mathrm{ab}}\end{array}$ & $\begin{array}{c}16.12 \\
(4.07)^{\mathrm{ab}}\end{array}$ & $\begin{array}{c}5.20 \\
(2.38)\end{array}$ \\
\hline 10. & Untreated control & $\begin{array}{l}74.49 \\
(8.40)\end{array}$ & $\begin{array}{l}84.29 \\
(8.96)^{\mathrm{c}}\end{array}$ & $\begin{array}{c}87.84 \\
(9.27)^{\mathrm{d}}\end{array}$ & $\begin{array}{c}86.65 \\
(9.00)^{\mathrm{e}}\end{array}$ & $\begin{array}{c}104.39 \\
(10.11)^{\mathrm{e}}\end{array}$ & $\begin{array}{c}104.85 \\
(10.05)^{f}\end{array}$ & $\begin{array}{l}94.49 \\
(9.63)^{\mathrm{f}}\end{array}$ & $\begin{array}{c}6.53 \\
(2.65)\end{array}$ \\
\hline & C.D@5\% & NS & 1.50 & 1.21 & 1.67 & 1.22 & 1.39 & 1.01 & NS \\
\hline & S.Em \pm & & 0.51 & 0.41 & 0.56 & 0.41 & 0.47 & 0.34 & \\
\hline & C.V $(\%)$ & & 12.24 & 10.33 & 14.50 & 9.67 & 13.69 & 10.84 & \\
\hline
\end{tabular}

Note: DBS - Day before spray. DAS - Days after spray.

Figures in the parentheses are $\sqrt{ }(x+0.5)$ transformed values.

Means followed by similar alphabets in the column do not differ significantly at $0.05 \%$ by DMRT. 
Table.2 Efficacy of insecticides and bio pesticides against thrips, Thrips tabaci (Lindeman) on Bt cotton ( $3^{\text {rd }}$ and $4^{\text {th }}$ spray)

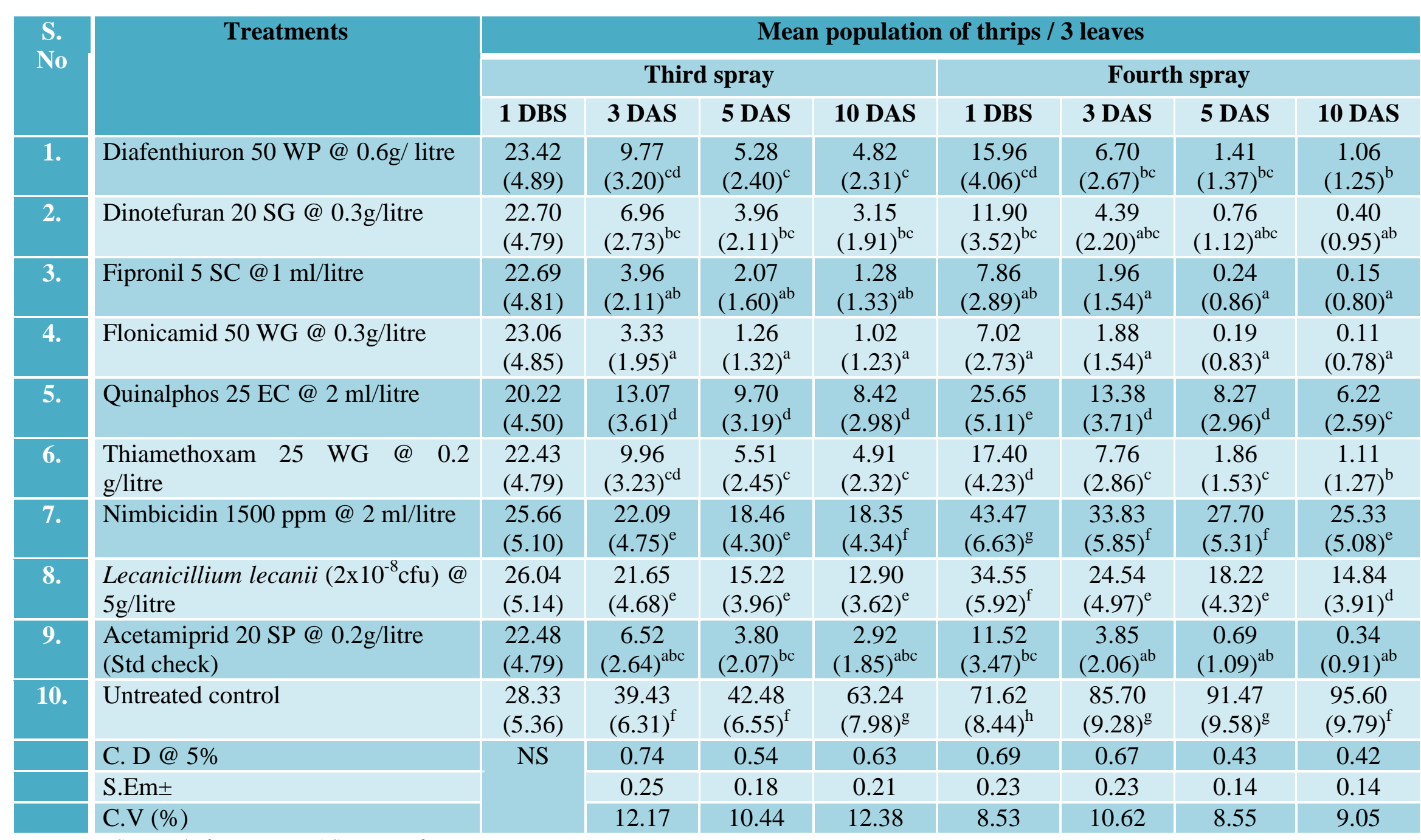

Note: DBS - Day before spray. DAS - Days after spray.

Figures in the parentheses are $\sqrt{ }(x+0.5)$ transformed values.

Means followed by similar alphabets in the column do not differ significantly at $0.05 \%$ by DMRT. 
Table.3 Efficacy of insecticides and bio pesticides against aphids Aphis gossypii (Glover) on Bt cotton $\left(3^{\text {rd }}\right.$ and $4^{\text {th }}$ spray)

\begin{tabular}{|c|c|c|c|c|c|c|c|c|c|}
\hline \multirow{3}{*}{$\begin{array}{l}\text { S. } \\
\text { No }\end{array}$} & \multirow[t]{3}{*}{ Treatments } & \multicolumn{8}{|c|}{ Mean population of aphids / 3 leaves } \\
\hline & & \multicolumn{4}{|c|}{ Third spray } & \multicolumn{4}{|c|}{ Fourth spray } \\
\hline & & $1 \mathrm{DBS}$ & 3 DAS & 5 DAS & 10 DAS & $1 \mathrm{DBS}$ & 3 DAS & 5 DAS & 10 DAS \\
\hline 1. & Diafenthiuron 50 WP @ 0.6g/ litre & $\begin{array}{l}15.80 \\
(4.03)\end{array}$ & $\begin{array}{c}9.26 \\
(3.12)^{\mathrm{ab}}\end{array}$ & $\begin{array}{c}5.96 \\
(2.54)^{\mathrm{ab}}\end{array}$ & $\begin{array}{c}4.60 \\
(2.26)^{\mathrm{ab}}\end{array}$ & $\begin{array}{c}16.00 \\
(4.06)^{a b}\end{array}$ & $\begin{array}{c}7.15 \\
(2.77)^{b}\end{array}$ & $\begin{array}{c}3.07 \\
(1.89)^{b}\end{array}$ & $\begin{array}{c}0.60 \\
(1.05)^{\mathrm{a}}\end{array}$ \\
\hline 2. & Dinotefuran 20 SG @ 0.3g/litre & $\begin{array}{l}14.60 \\
(3.85)\end{array}$ & $\begin{array}{c}7.91 \\
(2.90)^{\mathrm{a}}\end{array}$ & $\begin{array}{c}4.67 \\
(2.27)^{\mathrm{a}}\end{array}$ & $\begin{array}{c}3.67 \\
(2.04)^{\mathrm{a}}\end{array}$ & $\begin{array}{c}12.53 \\
(3.61)^{\mathrm{a}}\end{array}$ & $\begin{array}{c}3.03 \\
(1.88)^{\mathrm{ab}}\end{array}$ & $\begin{array}{c}0.56 \\
(1.02)^{\mathrm{a}}\end{array}$ & $\begin{array}{c}0.00 \\
(0.71)^{\mathrm{a}}\end{array}$ \\
\hline 3. & Fipronil 5 SC @1 ml/litre & $\begin{array}{l}15.80 \\
(4.02)\end{array}$ & $\begin{array}{c}8.87 \\
(3.06)^{\mathrm{a}}\end{array}$ & $\begin{array}{c}5.47 \\
(2.44)^{\mathrm{a}}\end{array}$ & $\begin{array}{c}4.32 \\
(2.19)^{\mathrm{a}}\end{array}$ & $\begin{array}{c}15.20 \\
(3.96)^{\mathrm{a}}\end{array}$ & $\begin{array}{c}6.53 \\
(2.65)^{\mathrm{ab}}\end{array}$ & $\begin{array}{c}2.47 \\
(1.72)^{b}\end{array}$ & $\begin{array}{c}0.00 \\
(0.71)^{\mathrm{a}}\end{array}$ \\
\hline 4. & Flonicamid 50 WG @ 0.3g/litre & $\begin{array}{l}15.33 \\
(3.97)\end{array}$ & $\begin{array}{c}7.33 \\
(2.80)^{\mathrm{a}}\end{array}$ & $\begin{array}{c}4.13 \\
(2.15)^{\mathrm{a}}\end{array}$ & $\begin{array}{c}3.20 \\
(1.92)^{\mathrm{a}}\end{array}$ & $\begin{array}{c}10.80 \\
(3.36)^{\mathrm{a}}\end{array}$ & $\begin{array}{c}2.60 \\
(1.76)^{\mathrm{a}}\end{array}$ & $\begin{array}{c}0.00 \\
(0.71)^{\mathrm{a}}\end{array}$ & $\begin{array}{c}0.00 \\
(0.71)^{\mathrm{a}}\end{array}$ \\
\hline 5. & Quinalphos 25 EC @ 2 ml/litre & $\begin{array}{l}15.23 \\
(3.92)\end{array}$ & $\begin{array}{c}11.17 \\
(3.41)^{\mathrm{abc}}\end{array}$ & $\begin{array}{c}8.84 \\
(3.05)^{b c}\end{array}$ & $\begin{array}{c}7.13 \\
(2.76)^{b c}\end{array}$ & $\begin{array}{c}22.67 \\
(4.81)^{b c}\end{array}$ & $\begin{array}{c}15.79 \\
(4.04)^{\mathrm{c}}\end{array}$ & $\begin{array}{c}9.47 \\
(3.15)^{\mathrm{c}}\end{array}$ & $\begin{array}{c}5.33 \\
(2.41)^{b}\end{array}$ \\
\hline 6. & $\begin{array}{l}\text { Thiamethoxam } \\
\text { g/litre }\end{array}$ & $\begin{array}{l}15.53 \\
(3.99)\end{array}$ & $\begin{array}{c}9.07 \\
(3.09)^{\mathrm{ab}}\end{array}$ & $\begin{array}{c}5.53 \\
(2.46)^{\mathrm{a}}\end{array}$ & $\begin{array}{c}4.45 \\
(2.22)^{\mathrm{a}}\end{array}$ & $\begin{array}{l}15.66 \\
(4.02)^{\mathrm{ab}}\end{array}$ & $\begin{array}{c}6.76 \\
(2.69)^{\mathrm{b}}\end{array}$ & $\begin{array}{c}2.60 \\
(1.75)^{b}\end{array}$ & $\begin{array}{c}0.47 \\
(0.98)^{\mathrm{a}}\end{array}$ \\
\hline 7. & Nimbicidin 1500 ppm @2 ml/litre & $\begin{array}{l}15.07 \\
(3.92)\end{array}$ & $\begin{array}{c}13.93 \\
(3.80)^{\mathrm{cd}}\end{array}$ & $\begin{array}{c}11.95 \\
(3.53)^{\mathrm{c}}\end{array}$ & $\begin{array}{c}11.13 \\
(3.41)^{\mathrm{d}}\end{array}$ & $\begin{array}{c}35.33 \\
(5.99)^{\mathrm{de}}\end{array}$ & $\begin{array}{c}29.20 \\
(5.45)^{d}\end{array}$ & $\begin{array}{c}21.73 \\
(4.71)^{\mathrm{e}}\end{array}$ & $\begin{array}{c}19.30 \\
(4.45)^{d}\end{array}$ \\
\hline 8. & $\begin{array}{l}\text { Lecanicillium lecanii }\left(2 \times 10^{-8} \mathrm{cfu}\right) @ \\
5 \mathrm{~g} / \text { litre }\end{array}$ & $\begin{array}{l}15.33 \\
(3.97)\end{array}$ & $\begin{array}{c}13.07 \\
(3.68)^{\mathrm{bc}}\end{array}$ & $\begin{array}{c}10.93 \\
(3.38)^{\mathrm{c}}\end{array}$ & $\begin{array}{c}9.07 \\
(3.09)^{\mathrm{cd}}\end{array}$ & $\begin{array}{l}29.53 \\
(5.48)^{\mathrm{cd}}\end{array}$ & $\begin{array}{c}22.71 \\
(4.81)^{\mathrm{cd}}\end{array}$ & $\begin{array}{l}15.87 \\
(4.04)^{d}\end{array}$ & $\begin{array}{c}9.06 \\
(3.09)^{\mathrm{c}}\end{array}$ \\
\hline 9. & $\begin{array}{l}\text { Acetamiprid } 20 \text { SP @ 0.2g/litre } \\
\text { (Std check) }\end{array}$ & $\begin{array}{l}15.57 \\
(4.01)\end{array}$ & $\begin{array}{c}7.83 \\
(2.88)^{\mathrm{a}}\end{array}$ & $\begin{array}{c}4.40 \\
(2.21)^{\mathrm{a}}\end{array}$ & $\begin{array}{c}3.53 \\
(2.01)^{\mathrm{a}}\end{array}$ & $\begin{array}{c}12.20 \\
(3.56)^{\mathrm{a}}\end{array}$ & $\begin{array}{c}2.93 \\
(1.85)^{\mathrm{ab}}\end{array}$ & $\begin{array}{c}0.00 \\
(0.71)^{\mathrm{a}}\end{array}$ & $\begin{array}{c}0.00 \\
(0.71)^{\mathrm{a}}\end{array}$ \\
\hline 10. & Untreated control & $\begin{array}{l}15.87 \\
(4.03)\end{array}$ & $\begin{array}{l}19.67 \\
(4.40)^{d}\end{array}$ & $\begin{array}{c}30.13 \\
(5.48)^{\mathrm{d}}\end{array}$ & $\begin{array}{l}52.47 \\
(7.24)^{\mathrm{e}}\end{array}$ & $\begin{array}{l}39.40 \\
(6.23)^{\mathrm{e}}\end{array}$ & $\begin{array}{l}45.62 \\
(6.65)^{e}\end{array}$ & $\begin{array}{l}51.73 \\
(7.18)^{f}\end{array}$ & $\begin{array}{c}59.67 \\
(7.73)^{\mathrm{e}}\end{array}$ \\
\hline & C.D@5\% & NS & 0.61 & 0.55 & 0.50 & 0.73 & 0.92 & 0.62 & 0.48 \\
\hline & S.Em \pm & & 0.21 & 0.19 & 0.17 & 0.25 & 0.31 & 0.21 & 0.16 \\
\hline & C.V (\%) & & 10.74 & 10.89 & 9.95 & 9.50 & 15.54 & 13.48 & 12.47 \\
\hline
\end{tabular}

Note: DBS - Day before spray. DAS - Days after spray.

Figures in the parentheses are $\sqrt{ }(x+0.5)$ transformed values.

Means followed by similar alphabets in the column do not differ significantly at $0.05 \%$ by DMRT. 
Table.4 Efficacy of insecticides and bio pesticides against jassids, Amrasca biguttula biguttula (Ishida) on Bt cotton ( $1^{\text {st }}$ and $2^{\text {nd }}$ spray)

\begin{tabular}{|c|c|c|c|c|c|c|c|c|c|}
\hline \multirow{3}{*}{$\begin{array}{l}\text { S. } \\
\text { No }\end{array}$} & \multirow[t]{3}{*}{ Treatments } & \multicolumn{8}{|c|}{ Mean population of jassids / 3 leaves } \\
\hline & & \multicolumn{4}{|c|}{ First spray } & \multicolumn{4}{|c|}{ Second spray } \\
\hline & & $1 \mathrm{DBS}$ & 3 DAS & 5 DAS & 10 DAS & $1 \mathrm{DBS}$ & 3 DAS & 5 DAS & 10 DAS \\
\hline 1. & Diafenthiuron50 WP @ 0.6g/ litre & $\begin{array}{c}0.67 \\
(1.08)\end{array}$ & $\begin{array}{c}0.26 \\
(0.87)^{\mathrm{ab}}\end{array}$ & $\begin{array}{c}0.13 \\
(0.79)^{\mathrm{ab}}\end{array}$ & $\begin{array}{c}0.07 \\
(0.76)^{\mathrm{ab}}\end{array}$ & $\begin{array}{c}0.40 \\
(0.95)^{\mathrm{a}}\end{array}$ & $\begin{array}{c}0.16 \\
(0.81)^{\mathrm{a}}\end{array}$ & $\begin{array}{c}0.09 \\
(0.77)^{\mathrm{ab}}\end{array}$ & $\begin{array}{c}0.00 \\
(0.71)\end{array}$ \\
\hline 2. & Dinotefuran 20 SG @ 0.3g/litre & $\begin{array}{c}0.69 \\
(1.09)\end{array}$ & $\begin{array}{c}0.21 \\
(0.84)^{\mathrm{a}}\end{array}$ & $\begin{array}{c}0.08 \\
(0.76)^{\mathrm{a}}\end{array}$ & $\begin{array}{c}0.00 \\
(0.71)^{\mathrm{a}}\end{array}$ & $\begin{array}{c}0.36 \\
(0.93)^{\mathrm{a}}\end{array}$ & $\begin{array}{c}0.13 \\
(0.79)^{\mathrm{a}}\end{array}$ & $\begin{array}{c}0.00 \\
(0.71)^{\mathrm{a}}\end{array}$ & $\begin{array}{c}0.00 \\
(0.71)\end{array}$ \\
\hline 3. & Fipronil 5 SC @1 ml/litre & $\begin{array}{c}0.68 \\
(1.08)\end{array}$ & $\begin{array}{c}0.34 \\
(0.91)^{\mathrm{ab}}\end{array}$ & $\begin{array}{c}0.19 \\
(0.83)^{\mathrm{ab}}\end{array}$ & $\begin{array}{c}0.13 \\
(0.79)^{\mathrm{ab}}\end{array}$ & $\begin{array}{c}0.61 \\
(1.05)^{\mathrm{ab}}\end{array}$ & $\begin{array}{c}0.30 \\
(0.89)^{\mathrm{ab}}\end{array}$ & $\begin{array}{c}0.21 \\
(0.84)^{\mathrm{ab}}\end{array}$ & $\begin{array}{c}0.00 \\
(0.71)\end{array}$ \\
\hline 4. & Flonicamid 50 WG @ 0.3g/litre & $\begin{array}{c}0.71 \\
(1.10)\end{array}$ & $\begin{array}{c}0.18 \\
(0.82)^{\mathrm{a}}\end{array}$ & $\begin{array}{c}0.00 \\
(0.71)^{\mathrm{a}}\end{array}$ & $\begin{array}{c}0.00 \\
(0.71)^{\mathrm{a}}\end{array}$ & $\begin{array}{c}0.33 \\
(0.91)^{\mathrm{a}}\end{array}$ & $\begin{array}{c}0.11 \\
(0.78)^{\mathrm{a}}\end{array}$ & $\begin{array}{c}0.00 \\
(0.71)^{\mathrm{a}}\end{array}$ & $\begin{array}{c}0.00 \\
(0.71)\end{array}$ \\
\hline 5. & Quinalphos 25 EC @ 2 ml/litre & $\begin{array}{c}0.70 \\
(1.09)\end{array}$ & $\begin{array}{c}0.55 \\
(1.02)^{\mathrm{bc}}\end{array}$ & $\begin{array}{c}0.39 \\
(0.94)^{\mathrm{bcd}}\end{array}$ & $\begin{array}{c}0.31 \\
(0.90)^{\mathrm{bc}}\end{array}$ & $\begin{array}{c}1.13 \\
(1.28)^{\mathrm{bc}}\end{array}$ & $\begin{array}{c}0.53 \\
(1.01)^{\mathrm{b}}\end{array}$ & $\begin{array}{c}0.37 \\
(0.93)^{b}\end{array}$ & $\begin{array}{c}0.00 \\
(0.71)\end{array}$ \\
\hline 6. & 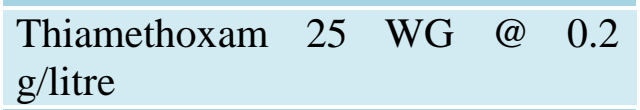 & $\begin{array}{c}0.66 \\
(1.08)\end{array}$ & $\begin{array}{c}0.33 \\
(0.91)^{\mathrm{ab}}\end{array}$ & $\begin{array}{c}0.21 \\
(0.84)^{\mathrm{abc}}\end{array}$ & $\begin{array}{c}0.17 \\
(0.82)^{\mathrm{ab}}\end{array}$ & $\begin{array}{c}0.57 \\
(1.03)^{\mathrm{a}}\end{array}$ & $\begin{array}{c}0.26 \\
(0.87)^{\mathrm{ab}}\end{array}$ & $\begin{array}{c}0.18 \\
(0.82)^{\mathrm{ab}}\end{array}$ & $\begin{array}{c}0.00 \\
(0.71)\end{array}$ \\
\hline 7. & Nimbicidin 1500 ppm @ 2 ml/litre & $\begin{array}{c}0.74 \\
(1.11)\end{array}$ & $\begin{array}{c}0.69 \\
(1.09)^{\mathrm{c}}\end{array}$ & $\begin{array}{c}0.62 \\
(1.06)^{\mathrm{de}}\end{array}$ & $\begin{array}{c}0.58 \\
(1.04)^{\mathrm{c}}\end{array}$ & $\begin{array}{c}1.50 \\
(1.41)^{\mathrm{cd}}\end{array}$ & $\begin{array}{c}1.33 \\
(1.35)^{\mathrm{c}}\end{array}$ & $\begin{array}{c}1.21 \\
(1.30)^{\mathrm{c}}\end{array}$ & $\begin{array}{c}0.00 \\
(0.71)\end{array}$ \\
\hline 8. & $\begin{array}{l}\left.\text { Lecanicillium lecanii ( } 2 \times 10^{-8} \mathrm{cfu}\right) \\
\text { @ } 5 \mathrm{~g} / \text { litre }\end{array}$ & $\begin{array}{c}0.74 \\
(1.11)\end{array}$ & $\begin{array}{c}0.65 \\
(1.07)^{\mathrm{c}}\end{array}$ & $\begin{array}{c}0.53 \\
(1.01)^{\text {cde }}\end{array}$ & $\begin{array}{c}0.47 \\
(0.99)^{\mathrm{c}}\end{array}$ & $\begin{array}{c}1.42 \\
(1.38)^{\mathrm{cd}}\end{array}$ & $\begin{array}{c}1.18 \\
(1.30)^{\mathrm{c}}\end{array}$ & $\begin{array}{c}1.02 \\
(1.23)^{\mathrm{c}}\end{array}$ & $\begin{array}{c}0.00 \\
(0.71)\end{array}$ \\
\hline 9. & $\begin{array}{l}\text { Acetamiprid 20 SP @ 0.2g/litre } \\
\text { (Std check) }\end{array}$ & $\begin{array}{c}0.68 \\
(1.09)\end{array}$ & $\begin{array}{c}0.29 \\
(0.89)^{\mathrm{ab}}\end{array}$ & $\begin{array}{c}0.13 \\
(0.79)^{\mathrm{ab}}\end{array}$ & $\begin{array}{c}0.09 \\
(0.77)^{\mathrm{ab}}\end{array}$ & $\begin{array}{c}0.40 \\
(0.95)^{\mathrm{a}}\end{array}$ & $\begin{array}{c}0.18 \\
(0.82)^{\mathrm{a}}\end{array}$ & $\begin{array}{c}0.11 \\
(0.78)^{\mathrm{ab}}\end{array}$ & $\begin{array}{c}0.00 \\
(0.71)\end{array}$ \\
\hline 10. & Untreated control & $\begin{array}{c}0.71 \\
(1.07)\end{array}$ & $\begin{array}{c}0.80 \\
(1.12)^{\mathrm{c}}\end{array}$ & $\begin{array}{c}0.87 \\
(1.15)^{\mathrm{e}}\end{array}$ & $\begin{array}{c}1.31 \\
(1.33)^{d}\end{array}$ & $\begin{array}{c}2.01 \\
(1.55)^{d}\end{array}$ & $\begin{array}{c}2.09 \\
(1.59)^{\mathrm{d}}\end{array}$ & $\begin{array}{c}2.47 \\
(1.70)^{\mathrm{d}}\end{array}$ & $\begin{array}{c}0.07 \\
(0.75)\end{array}$ \\
\hline & C.D@5\% & NS & 0.15 & 0.17 & 0.16 & 0.23 & 0.18 & 0.21 & NS \\
\hline & S.Em \pm & & 0.05 & 0.06 & 0.06 & 0.08 & 0.06 & 0.07 & \\
\hline & C.V $(\%)$ & & 9.21 & 10.43 & 10.82 & 11.91 & 10.27 & 12.56 & \\
\hline
\end{tabular}

Note: DBS - Day before spray. DAS - Days after spray.

Figures in the parentheses are $\sqrt{ }(x+0.5)$ transformed values.

Means followed by similar alphabets in the column do not differ significantly at $0.05 \%$ by DMRT. 
Table.5 Efficacy of insecticides and bio pesticides against jassids, Amrasca biguttula biguttula (Ishida) on Bt cotton ( $3^{\text {rd }}$ and $4^{\text {th }}$ spray)

\begin{tabular}{|c|c|c|c|c|c|c|c|c|c|}
\hline \multirow{3}{*}{$\begin{array}{l}\text { S. } \\
\text { No }\end{array}$} & \multirow[t]{3}{*}{ Treatments } & \multicolumn{8}{|c|}{ Mean population of jassids / 3 leaves } \\
\hline & & \multicolumn{4}{|c|}{ Third spray } & \multicolumn{4}{|c|}{ Fourth spray } \\
\hline & & 1 DBS & 3 DAS & 5 DAS & 10 DAS & 1 DBS & 3 DAS & 5 DAS & 10 DAS \\
\hline 1. & Diafenthiuron 50 WP @ 0.6g/ litre & $\begin{array}{c}0.43 \\
(0.96)\end{array}$ & $\begin{array}{c}0.23 \\
(0.85)^{\mathrm{ab}}\end{array}$ & $\begin{array}{c}0.13 \\
(0.79)^{\mathrm{ab}}\end{array}$ & $\begin{array}{c}0.07 \\
(0.75)^{\mathrm{a}}\end{array}$ & $\begin{array}{c}0.61 \\
(1.05)^{\mathrm{ab}}\end{array}$ & $\begin{array}{c}0.30 \\
(0.89)^{\mathrm{a}}\end{array}$ & $\begin{array}{c}0.18 \\
(0.82)^{\mathrm{a}}\end{array}$ & $\begin{array}{c}0.09 \\
(0.77)^{\mathrm{a}}\end{array}$ \\
\hline 2. & Dinotefuran 20 SG @ 0.3g/litre & $\begin{array}{c}0.38 \\
(0.93)\end{array}$ & $\begin{array}{c}0.19 \\
(0.83)^{\mathrm{ab}}\end{array}$ & $\begin{array}{c}0.07 \\
(0.76)^{\mathrm{a}}\end{array}$ & $\begin{array}{c}0.00 \\
(0.71)^{\mathrm{a}}\end{array}$ & $\begin{array}{c}0.48 \\
(0.99)^{\mathrm{a}}\end{array}$ & $\begin{array}{c}0.24 \\
(0.86)^{\mathrm{a}}\end{array}$ & $\begin{array}{c}0.13 \\
(0.79)^{\mathrm{a}}\end{array}$ & $\begin{array}{c}0.00 \\
(0.71)^{\mathrm{a}}\end{array}$ \\
\hline 3. & Fipronil 5 SC @1 ml/litre & $\begin{array}{c}0.55 \\
(1.02)\end{array}$ & $\begin{array}{c}0.30 \\
(0.89)^{\mathrm{ab}}\end{array}$ & $\begin{array}{c}0.21 \\
(0.84)^{\mathrm{ab}}\end{array}$ & $\begin{array}{c}0.14 \\
(0.80)^{\mathrm{ab}}\end{array}$ & $\begin{array}{c}0.83 \\
(1.15)^{\mathrm{ab}}\end{array}$ & $\begin{array}{c}0.41 \\
(0.95)^{\mathrm{ab}}\end{array}$ & $\begin{array}{c}0.27 \\
(0.88)^{\mathrm{ab}}\end{array}$ & $\begin{array}{c}0.21 \\
(0.84)^{\mathrm{a}}\end{array}$ \\
\hline 4. & Flonicamid 50 WG @ 0.3g/litre & $\begin{array}{c}0.34 \\
(0.91)\end{array}$ & $\begin{array}{c}0.15 \\
(0.81)^{\mathrm{a}}\end{array}$ & $\begin{array}{c}0.00 \\
(0.71)^{\mathrm{a}}\end{array}$ & $\begin{array}{c}0.00 \\
(0.71)^{\mathrm{a}}\end{array}$ & $\begin{array}{c}0.43 \\
(0.96)^{\mathrm{a}}\end{array}$ & $\begin{array}{c}0.18 \\
(0.82)^{\mathrm{a}}\end{array}$ & $\begin{array}{c}0.00 \\
(0.71)^{\mathrm{a}}\end{array}$ & $\begin{array}{c}0.00 \\
(0.71)^{\mathrm{a}}\end{array}$ \\
\hline 5. & Quinalphos 25 EC @ 2 ml/litre & $\begin{array}{c}0.63 \\
(1.06)\end{array}$ & $\begin{array}{c}0.48 \\
(0.99)^{\mathrm{bc}}\end{array}$ & $\begin{array}{c}0.39 \\
(0.94)^{\mathrm{bc}}\end{array}$ & $\begin{array}{c}0.32 \\
(0.90)^{\mathrm{abc}}\end{array}$ & $\begin{array}{l}1.27 \\
\left(1.32^{\mathrm{bc}}\right.\end{array}$ & $\begin{array}{c}0.88 \\
(1.17)^{b}\end{array}$ & $\begin{array}{c}0.57 \\
(1.04)^{b}\end{array}$ & $\begin{array}{c}0.36 \\
(0.93)^{\mathrm{ab}}\end{array}$ \\
\hline 6. & 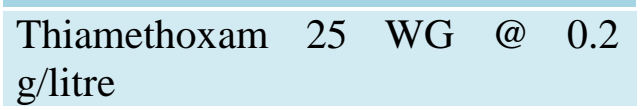 & $\begin{array}{c}0.53 \\
(1.02)\end{array}$ & $\begin{array}{c}0.27 \\
(0.87)^{\mathrm{ab}}\end{array}$ & $\begin{array}{c}0.18 \\
(0.82)^{\mathrm{ab}}\end{array}$ & $\begin{array}{c}0.12 \\
(0.79)^{\mathrm{ab}}\end{array}$ & $\begin{array}{c}0.81 \\
(1.14)^{\mathrm{ab}}\end{array}$ & $\begin{array}{c}0.39 \\
(0.94)^{\mathrm{ab}}\end{array}$ & $\begin{array}{c}0.24 \\
(0.86)^{\mathrm{ab}}\end{array}$ & $\begin{array}{c}0.17 \\
(0.82)^{\mathrm{a}}\end{array}$ \\
\hline 7. & Nimbicidin 1500 ppm @ 2 ml/litre & $\begin{array}{c}0.73 \\
(1.11)\end{array}$ & $\begin{array}{c}0.65 \\
(1.07)^{\mathrm{c}}\end{array}$ & $\begin{array}{c}0.59 \\
(1.04)^{\mathrm{c}}\end{array}$ & $\begin{array}{c}0.54 \\
(1.02)^{\mathrm{c}}\end{array}$ & $\begin{array}{c}2.52 \\
(1.74)^{d}\end{array}$ & $\begin{array}{c}2.09 \\
(1.61)^{\mathrm{c}}\end{array}$ & $\begin{array}{c}1.75 \\
(1.50)^{\mathrm{d}}\end{array}$ & $\begin{array}{c}1.40 \\
(1.37)^{\mathrm{c}}\end{array}$ \\
\hline 8. & $\begin{array}{l}\left.\text { Lecanicillium lecanii ( } 2 \times 10^{-8} \mathrm{cfu}\right) \\
\text { @ } 5 \mathrm{~g} / \text { litre }\end{array}$ & $\begin{array}{c}0.67 \\
(1.08)\end{array}$ & $\begin{array}{c}0.62 \\
(1.06)^{\mathrm{c}}\end{array}$ & $\begin{array}{c}0.52 \\
(1.01)^{\mathrm{c}}\end{array}$ & $\begin{array}{c}0.44 \\
(0.97)^{\mathrm{bc}}\end{array}$ & $\begin{array}{c}2.10 \\
(1.61)^{\mathrm{cd}}\end{array}$ & $\begin{array}{c}1.73 \\
(1.49)^{\mathrm{c}}\end{array}$ & $\begin{array}{c}1.20 \\
(1.30)^{\mathrm{c}}\end{array}$ & $\begin{array}{c}1.01 \\
(1.23)^{\mathrm{bc}}\end{array}$ \\
\hline 9. & $\begin{array}{l}\text { Acetamiprid } 20 \text { SP @ 0.2g/litre } \\
\text { (Std check) }\end{array}$ & $\begin{array}{c}0.51 \\
(1.00)\end{array}$ & $\begin{array}{c}0.24 \\
(0.86)^{\mathrm{ab}}\end{array}$ & $\begin{array}{c}0.15 \\
(0.81)^{\mathrm{ab}}\end{array}$ & $\begin{array}{c}0.06 \\
(0.75)^{\mathrm{a}}\end{array}$ & $\begin{array}{c}0.67 \\
(1.08)^{\mathrm{ab}}\end{array}$ & $\begin{array}{c}0.33 \\
(0.91)^{\mathrm{a}}\end{array}$ & $\begin{array}{c}0.20 \\
(0.84)^{\mathrm{a}}\end{array}$ & $\begin{array}{c}0.13 \\
(0.79)^{\mathrm{a}}\end{array}$ \\
\hline 10. & Untreated control & $\begin{array}{c}0.86 \\
(1.16)\end{array}$ & $\begin{array}{l}1.45 \\
(1.37)^{\mathrm{d}}\end{array}$ & $\begin{array}{c}1.92 \\
(1.54)^{d}\end{array}$ & $\begin{array}{c}1.73 \\
(1.47)^{d}\end{array}$ & $\begin{array}{c}4.03 \\
(2.09)^{\mathrm{e}}\end{array}$ & $\begin{array}{c}4.55 \\
(2.24)^{\mathrm{d}}\end{array}$ & $\begin{array}{c}4.20 \\
(2.15)^{\mathrm{e}}\end{array}$ & $\begin{array}{c}4.08 \\
(2.09)^{\mathrm{d}}\end{array}$ \\
\hline & C.D@5\% & NS & 0.16 & 0.15 & 0.19 & 0.31 & 0.23 & 0.19 & 0.30 \\
\hline & S.Em \pm & & 0.05 & 0.05 & 0.06 & 0.10 & 0.08 & 0.06 & 0.10 \\
\hline & C.V $(\%)$ & & 9.82 & 9.41 & 12.21 & 13.56 & 11.24 & 10.19 & 17.21 \\
\hline
\end{tabular}

Note: DBS - Day before spray. DAS - Days after spray.

Figures in the parentheses are $\sqrt{ }(x+0.5)$ transformed values

Means followed by similar alphabets in the column do not differ significantly at $0.05 \%$ by DMRT. 
Table.6 Efficacy of insecticides and bio pesticides against whitefly, Bemisia tabaci (Gennadius) on Bt cotton $\left(1^{\text {st }}\right.$ and $2^{\text {nd }}$ spray)

\begin{tabular}{|c|c|c|c|c|c|c|c|c|c|}
\hline \multirow{3}{*}{$\begin{array}{c}\text { S. } \\
\text { No }\end{array}$} & \multirow[t]{3}{*}{ Treatments } & \multicolumn{8}{|c|}{ Mean population of whiteflies / 3 leaves } \\
\hline & & \multicolumn{4}{|c|}{ First spray } & \multicolumn{4}{|c|}{ Second spray } \\
\hline & & $1 \mathrm{DBS}$ & 3 DAS & 5 DAS & 10 DAS & $1 \mathrm{DBS}$ & 3 DAS & 5 DAS & 10 DAS \\
\hline 1. & Diafenthiuron50 WP @ 0.6g/ litre & $\begin{array}{c}0.32 \\
(0.90)\end{array}$ & $\begin{array}{c}0.00 \\
(0.71)^{\mathrm{a}}\end{array}$ & $\begin{array}{c}0.00 \\
(0.71)^{\mathrm{a}}\end{array}$ & $\begin{array}{c}0.00 \\
(0.71)^{\mathrm{a}}\end{array}$ & $\begin{array}{c}0.16 \\
(0.81)^{\mathrm{a}}\end{array}$ & $\begin{array}{c}0.00 \\
(0.71)^{\mathrm{a}}\end{array}$ & $\begin{array}{c}0.00 \\
(0.71)^{\mathrm{a}}\end{array}$ & $\begin{array}{c}0.00 \\
(0.71)\end{array}$ \\
\hline 2. & Dinotefuran 20 SG @ 0.3g/litre & $\begin{array}{c}0.32 \\
(0.90)\end{array}$ & $\begin{array}{c}0.00 \\
(0.71)^{\mathrm{a}}\end{array}$ & $\begin{array}{c}0.00 \\
(0.71)^{\mathrm{a}}\end{array}$ & $\begin{array}{c}0.00 \\
(0.71)^{\mathrm{a}}\end{array}$ & $\begin{array}{c}0.13 \\
(0.79)^{\mathrm{a}}\end{array}$ & $\begin{array}{c}0.00 \\
(0.71)^{\mathrm{a}}\end{array}$ & $\begin{array}{c}0.00 \\
(0.71)^{\mathrm{a}}\end{array}$ & $\begin{array}{c}0.00 \\
(0.71)\end{array}$ \\
\hline 3. & Fipronil 5 SC @1 ml/litre & $\begin{array}{c}0.37 \\
(0.93)\end{array}$ & $\begin{array}{c}0.11 \\
(0.78)^{\mathrm{ab}}\end{array}$ & $\begin{array}{c}0.00 \\
(0.71)^{\mathrm{a}}\end{array}$ & $\begin{array}{c}0.07 \\
(0.76)^{\mathrm{a}}\end{array}$ & $\begin{array}{c}0.20 \\
(0.84)^{\mathrm{ab}}\end{array}$ & $\begin{array}{c}0.09 \\
(0.77)^{\mathrm{ab}}\end{array}$ & $\begin{array}{c}0.00 \\
(0.71)^{\mathrm{a}}\end{array}$ & $\begin{array}{c}0.00 \\
(0.71)\end{array}$ \\
\hline 4. & Flonicamid 50 WG @ 0.3g/litre & $\begin{array}{c}0.38 \\
(0.94)\end{array}$ & $\begin{array}{c}0.00 \\
(0.71)^{\mathrm{a}}\end{array}$ & $\begin{array}{c}0.00 \\
(0.71)^{\mathrm{a}}\end{array}$ & $\begin{array}{c}0.00 \\
(0.71)^{\mathrm{a}}\end{array}$ & $\begin{array}{c}0.08 \\
(0.78)^{\mathrm{a}}\end{array}$ & $\begin{array}{c}0.00 \\
(0.71)^{\mathrm{a}}\end{array}$ & $\begin{array}{c}0.00 \\
(0.71)^{\mathrm{a}}\end{array}$ & $\begin{array}{c}0.00 \\
(0.71)\end{array}$ \\
\hline 5. & Quinalphos 25 EC @ 2 ml/litre & $\begin{array}{c}0.43 \\
(0.97)\end{array}$ & $\begin{array}{c}0.34 \\
(0.91)^{\mathrm{bc}}\end{array}$ & $\begin{array}{c}0.26 \\
(0.87)^{b}\end{array}$ & $\begin{array}{c}0.15 \\
(0.80)^{\mathrm{ab}}\end{array}$ & $\begin{array}{c}0.43 \\
(0.96)^{b c}\end{array}$ & $\begin{array}{c}0.24 \\
(0.86)^{\mathrm{bc}}\end{array}$ & $\begin{array}{c}0.17 \\
(0.82)^{\mathrm{ab}}\end{array}$ & $\begin{array}{c}0.00 \\
(0.71)\end{array}$ \\
\hline 6. & $\begin{array}{l}\text { Thiamethoxam } 25 \text { WG @ } 0.2 \\
\text { g/litre }\end{array}$ & $\begin{array}{c}0.47 \\
(0.98)\end{array}$ & $\begin{array}{c}0.11 \\
(0.78)^{\mathrm{ab}}\end{array}$ & $\begin{array}{c}0.00 \\
(0.71)^{\mathrm{a}}\end{array}$ & $\begin{array}{c}0.00 \\
(0.71)^{\mathrm{a}}\end{array}$ & $\begin{array}{c}0.21 \\
(0.84)^{\mathrm{ab}}\end{array}$ & $\begin{array}{c}0.09 \\
(0.77)^{\mathrm{ab}}\end{array}$ & $\begin{array}{c}0.00 \\
(0.71)^{\mathrm{a}}\end{array}$ & $\begin{array}{c}0.00 \\
(0.71)\end{array}$ \\
\hline 7. & Nimbicidin 1500 ppm @ 2 ml/litre & $\begin{array}{c}0.43 \\
(0.96)\end{array}$ & $\begin{array}{c}0.41 \\
(0.96)^{\mathrm{c}}\end{array}$ & $\begin{array}{c}0.34 \\
(0.92)^{\mathrm{bc}}\end{array}$ & $\begin{array}{c}0.30 \\
(0.89)^{\mathrm{bc}}\end{array}$ & $\begin{array}{c}0.51 \\
(1.00)^{\mathrm{cd}}\end{array}$ & $\begin{array}{c}0.35 \\
(0.92)^{\mathrm{c}}\end{array}$ & $\begin{array}{c}0.30 \\
(0.89)^{\mathrm{bc}}\end{array}$ & $\begin{array}{c}0.09 \\
(0.77)\end{array}$ \\
\hline 8. & $\begin{array}{l}\text { Lecanicillium lecanii }\left(2 \times 10^{-8} \mathrm{cfu}\right) \\
\text { @ } 5 \mathrm{~g} / \text { litre }\end{array}$ & $\begin{array}{c}0.46 \\
(0.98)\end{array}$ & $\begin{array}{c}0.40 \\
(0.95)^{\mathrm{c}}\end{array}$ & $\begin{array}{c}0.32 \\
(0.91)^{\mathrm{bc}}\end{array}$ & $\begin{array}{c}0.27 \\
(0.87)^{\mathrm{bc}}\end{array}$ & $\begin{array}{c}0.49 \\
(0.99)^{\mathrm{cd}}\end{array}$ & $\begin{array}{c}0.31 \\
(0.90)^{\mathrm{bc}}\end{array}$ & $\begin{array}{c}0.27 \\
(0.87)^{\mathrm{bc}}\end{array}$ & $\begin{array}{c}0.07 \\
(0.75)\end{array}$ \\
\hline 9. & $\begin{array}{l}\text { Acetamiprid } 20 \text { SP @ 0.2g/litre } \\
\text { (Std check) }\end{array}$ & $\begin{array}{c}0.39 \\
(0.95)\end{array}$ & $\begin{array}{l}0.07 \\
(0.75)^{\mathrm{a}}\end{array}$ & $\begin{array}{c}0.00 \\
(0.71)^{\mathrm{a}}\end{array}$ & $\begin{array}{c}0.00 \\
(0.71)^{\mathrm{a}}\end{array}$ & $\begin{array}{c}0.16 \\
(0.81)^{\mathrm{a}}\end{array}$ & $\begin{array}{c}0.00 \\
(0.71)^{\mathrm{a}}\end{array}$ & $\begin{array}{c}0.00 \\
(0.71)^{\mathrm{a}}\end{array}$ & $\begin{array}{c}0.00 \\
(0.71)\end{array}$ \\
\hline 10. & Untreated control & $\begin{array}{c}0.38 \\
(0.92)\end{array}$ & $\begin{array}{c}0.49 \\
(0.98)^{\mathrm{c}}\end{array}$ & $\begin{array}{c}0.56 \\
(1.01)^{\mathrm{c}}\end{array}$ & $\begin{array}{c}0.42 \\
(0.94)^{\mathrm{c}}\end{array}$ & $\begin{array}{c}0.60 \\
(1.04)^{\mathrm{d}}\end{array}$ & $\begin{array}{c}0.41 \\
(0.94)^{\mathrm{c}}\end{array}$ & $\begin{array}{c}0.45 \\
(0.96)^{\mathrm{c}}\end{array}$ & $\begin{array}{c}0.12 \\
(0.79)\end{array}$ \\
\hline & C.D@5\% & NS & 0.13 & 0.13 & 0.13 & 0.14 & 0.13 & 0.12 & NS \\
\hline & S.Em \pm & & 0.05 & 0.04 & 0.04 & 0.05 & 0.04 & 0.04 & \\
\hline & C.V $(\%)$ & & 9.37 & 9.70 & 9.80 & 9.47 & 9.44 & 9.75 & \\
\hline
\end{tabular}

Note: DBS - Day before spray. DAS - Days after spray.

Figures in the parentheses are $\sqrt{ }(x+0.5)$ transformed values.

Means followed by similar alphabets in the column do not differ significantly at $0.05 \%$ by DMRT. 
Table.7 Bio efficacy of insecticides and bio pesticides against whitefly, Bemisia tabaci (Gennadius) on Bt cotton $\left(3^{\text {rd }}\right.$ and $4^{\text {th }}$ spray)

\begin{tabular}{|c|c|c|c|c|c|c|c|c|c|}
\hline \multirow{3}{*}{$\begin{array}{l}\text { S. } \\
\text { No }\end{array}$} & \multirow[t]{3}{*}{ Treatments } & \multicolumn{8}{|c|}{ Mean population of whiteflies / 3 leaves } \\
\hline & & \multicolumn{4}{|c|}{ Third spray } & \multicolumn{4}{|c|}{ Fourth spray } \\
\hline & & 1 DBS & 3 DAS & 5 DAS & 10 DAS & $1 \mathrm{DBS}$ & 3 DAS & 5 DAS & 10 DAS \\
\hline 1. & Diafenthiuron50 WP @ 0.6g/ litre & $\begin{array}{c}0.38 \\
(0.94)\end{array}$ & $\begin{array}{c}0.08 \\
(0.76)^{\mathrm{a}}\end{array}$ & $\begin{array}{c}0.00 \\
(0.71)^{\mathrm{a}}\end{array}$ & $\begin{array}{c}0.00 \\
(0.71)^{\mathrm{a}}\end{array}$ & $\begin{array}{c}1.27 \\
(1.33)^{\mathrm{ab}}\end{array}$ & $\begin{array}{c}0.38 \\
(0.94)^{\mathrm{a}}\end{array}$ & $\begin{array}{c}0.18 \\
(0.82)^{\mathrm{a}}\end{array}$ & $\begin{array}{c}0.04 \\
(0.73)^{\mathrm{a}}\end{array}$ \\
\hline 2. & Dinotefuran20 SG @ 0.3g/litre & $\begin{array}{c}0.33 \\
(0.91)\end{array}$ & $\begin{array}{c}0.00 \\
(0.71)^{\mathrm{a}}\end{array}$ & $\begin{array}{c}0.00 \\
(0.71)^{\mathrm{a}}\end{array}$ & $\begin{array}{c}0.00 \\
(0.71)^{\mathrm{a}}\end{array}$ & $\begin{array}{c}0.93 \\
(1.18)^{\mathrm{a}}\end{array}$ & $\begin{array}{c}0.29 \\
(0.89)^{\mathrm{a}}\end{array}$ & $\begin{array}{c}0.13 \\
(0.79)^{\mathrm{a}}\end{array}$ & $\begin{array}{c}0.00 \\
(0.71)^{\mathrm{a}}\end{array}$ \\
\hline 3. & Fipronil5 SC @1 ml/litre & $\begin{array}{c}0.43 \\
(0.97)\end{array}$ & $\begin{array}{c}0.14 \\
(0.80)^{\mathrm{ab}}\end{array}$ & $\begin{array}{c}0.00 \\
(0.71)^{\mathrm{a}}\end{array}$ & $\begin{array}{c}0.00 \\
(0.71)^{\mathrm{a}}\end{array}$ & $\begin{array}{c}1.39 \\
(1.37)^{\mathrm{ab}}\end{array}$ & $\begin{array}{c}0.53 \\
(1.02)^{\mathrm{a}}\end{array}$ & $\begin{array}{c}0.28 \\
(0.88)^{\mathrm{a}}\end{array}$ & $\begin{array}{c}0.18 \\
(0.82)^{\mathrm{a}}\end{array}$ \\
\hline 4. & Flonicamid 50WG @ 0.3g/litre & $\begin{array}{c}0.33 \\
(0.91)\end{array}$ & $\begin{array}{c}0.00 \\
(0.71)^{\mathrm{a}}\end{array}$ & $\begin{array}{c}0.00 \\
(0.71)^{\mathrm{a}}\end{array}$ & $\begin{array}{c}0.00 \\
(0.71)^{\mathrm{a}}\end{array}$ & $\begin{array}{c}0.80 \\
(1.13)^{\mathrm{a}}\end{array}$ & $\begin{array}{c}0.24 \\
(0.85)^{\mathrm{a}}\end{array}$ & $\begin{array}{c}0.07 \\
(0.75)^{\mathrm{a}}\end{array}$ & $\begin{array}{c}0.00 \\
(0.71)^{\mathrm{a}}\end{array}$ \\
\hline 5. & Quinalphos 25 EC @ 2 ml/litre & $\begin{array}{c}0.43 \\
(0.96)\end{array}$ & $\begin{array}{c}0.30 \\
(0.90)^{b c}\end{array}$ & $\begin{array}{c}0.19 \\
(0.83)^{\mathrm{ab}}\end{array}$ & $\begin{array}{c}0.12 \\
(0.79)^{\mathrm{ab}}\end{array}$ & $\begin{array}{c}1.84 \\
(1.53)^{b c}\end{array}$ & $\begin{array}{c}1.29 \\
(1.34)^{b c}\end{array}$ & $\begin{array}{c}0.82 \\
(1.15)^{\mathrm{bc}}\end{array}$ & $\begin{array}{c}0.47 \\
(0.98)^{\mathrm{ab}}\end{array}$ \\
\hline 6. & $\begin{array}{l}\text { Thiamethoxam } 25 \text { WG @ } 0.2 \\
\text { g/litre }\end{array}$ & $\begin{array}{c}0.41 \\
(0.95)\end{array}$ & $\begin{array}{c}0.16 \\
(0.81)^{\mathrm{ab}}\end{array}$ & $\begin{array}{c}0.00 \\
(0.71)^{\mathrm{a}}\end{array}$ & $\begin{array}{c}0.00 \\
(0.71)^{\mathrm{a}}\end{array}$ & $\begin{array}{c}1.43 \\
(1.39)^{\mathrm{ab}}\end{array}$ & $\begin{array}{c}0.59 \\
(1.05)^{\mathrm{ab}}\end{array}$ & $\begin{array}{c}0.33 \\
(0.91)^{\mathrm{ab}}\end{array}$ & $\begin{array}{c}0.24 \\
(0.86)^{\mathrm{a}}\end{array}$ \\
\hline 7. & Nimbicidin 1500 ppm @2 ml/litre & $\begin{array}{c}0.46 \\
(0.98)\end{array}$ & $\begin{array}{c}0.41 \\
(0.95)^{\mathrm{cd}}\end{array}$ & $\begin{array}{c}0.37 \\
(0.93)^{b}\end{array}$ & $\begin{array}{c}0.34 \\
(0.92)^{b}\end{array}$ & $\begin{array}{c}2.69 \\
(1.78)^{\mathrm{cd}}\end{array}$ & $\begin{array}{c}2.27 \\
(1.65)^{\mathrm{de}}\end{array}$ & $\begin{array}{c}1.60 \\
(1.44)^{d}\end{array}$ & $\begin{array}{c}1.27 \\
(1.33)^{\mathrm{c}}\end{array}$ \\
\hline 8. & $\begin{array}{l}\text { Lecanicillium lecanii }\left(2 \times 10^{-8} \mathrm{cfu}\right) \\
\text { @ } 5 \mathrm{~g} / \text { litre }\end{array}$ & $\begin{array}{c}0.46 \\
(0.98)\end{array}$ & $\begin{array}{c}0.39 \\
(0.95)^{\mathrm{cd}}\end{array}$ & $\begin{array}{c}0.34 \\
(0.92)^{b}\end{array}$ & $\begin{array}{c}0.27 \\
(0.88)^{b}\end{array}$ & $\begin{array}{c}2.57 \\
(1.75)^{\mathrm{cd}}\end{array}$ & $\begin{array}{c}1.90 \\
(1.55)^{\mathrm{cd}}\end{array}$ & $\begin{array}{c}1.32 \\
(1.34)^{\mathrm{cd}}\end{array}$ & $\begin{array}{l}1.00 \\
(1.22)^{b c}\end{array}$ \\
\hline 9. & $\begin{array}{l}\text { Acetamiprid } 20 \text { SP @ 0.2g/litre } \\
\text { (Std check) }\end{array}$ & $\begin{array}{c}0.40 \\
(0.95)\end{array}$ & $\begin{array}{c}0.11 \\
(0.78)^{\mathrm{ab}}\end{array}$ & $\begin{array}{c}0.00 \\
(0.71)^{\mathrm{a}}\end{array}$ & $\begin{array}{c}0.00 \\
(0.71)^{\mathrm{a}}\end{array}$ & $\begin{array}{c}1.33 \\
(1.35)^{\mathrm{ab}}\end{array}$ & $\begin{array}{c}0.43 \\
(0.96)^{\mathrm{a}}\end{array}$ & $\begin{array}{c}0.22 \\
(0.85)^{\mathrm{a}}\end{array}$ & $\begin{array}{c}0.09 \\
(0.77)^{\mathrm{a}}\end{array}$ \\
\hline 10. & Untreated control & $\begin{array}{c}0.48 \\
(0.99)\end{array}$ & $\begin{array}{c}0.64 \\
(1.05)^{d}\end{array}$ & $\begin{array}{c}1.31 \\
(1.32)^{\mathrm{c}}\end{array}$ & $\begin{array}{c}2.78 \\
(1.79)^{\mathrm{c}}\end{array}$ & $\begin{array}{c}3.11 \\
(1.87)^{\mathrm{d}}\end{array}$ & $\begin{array}{c}3.32 \\
(1.92)^{\mathrm{e}}\end{array}$ & $\begin{array}{c}3.06 \\
(1.87)^{\mathrm{e}}\end{array}$ & $\begin{array}{c}2.23 \\
(1.61)^{\mathrm{d}}\end{array}$ \\
\hline & C.D@ $5 \%$ & NS & 0.13 & 0.16 & 0.16 & 0.31 & 0.29 & 0.24 & 0.27 \\
\hline & S.Em \pm & & 0.05 & 0.05 & 0.06 & 0.10 & 0.10 & 0.08 & 0.09 \\
\hline & C.V $(\%)$ & & 9.26 & 10.94 & 10.98 & 12.32 & 13.73 & 12.69 & 16.10 \\
\hline
\end{tabular}

Note: DBS - Day before spray. DAS - Days after spray.

Figures in the parentheses are $\sqrt{ }(x+0.5)$ transformed values.

Means followed by similar alphabets in the column do not differ significantly at $0.05 \%$ by DMRT 
The next best treatments were acetamiprid 20 SP, thiamethoxam $25 \mathrm{WG}$ and fipronil $5 \mathrm{SC}$ were found superior and on par with each other with the white fly population of $(0.07$, $0.11,0.11$ whiteflies / 3 leaves). In all the spray the trend of supremacy of the treatments over whiteflies were not changed (Table 6 and 7 ). The results of the present investigation concludes that the flonicamid $50 \mathrm{WG}$, dinotefuran $20 \mathrm{SG}$ and diafenthiuron $50 \mathrm{WP}$ found highly effective against whiteflies, followed by acetamiprid 20 SP, thiamethoxam $25 \mathrm{WG}$ and fipronil $5 \mathrm{SC}$.

Present findings are in close agreement with the results of Ghelani et al., (2014) they also observed that the flonicamid caused significantly maximum mortality of whiteflies $(71.47 \%)$ and it was statistically at par with acetamiprid (69.83\%) and imidacloprid $(66.17 \%)$ followed by dinotefuron $(63.06 \%)$ thiamethoxam (62.76 \%). Razaq et al., (2005) also observed minimum number of whiteflies (5.39 whiteflies / leaf) in plots treated with diafenthiuron, acetamiprid (5.85 whiteflies / leaf) and imidacloprid (6.03 whiteflies / leaf) at 24 hours after application of insecticides.

In the present study at ten days after $2^{\text {nd }}$ spray and one day before $3^{\text {rd }}$ spray the pest population was statistically uniform in all the treatments because of rainfall in the respective week, all the pests were washed away and efficacy of the previous spray was not present to affect the pest population.

Overall investigation accomplished that the treatment flonicamid emerged as most superior and promising insecticide over rest of the treatments against all the sucking pests viz., thrips, aphids, jassids and whiteflies followed by fipronil $5 \mathrm{SC}$, acetamiprid $20 \mathrm{SP}$, dinotefuron $20 \mathrm{SG}$, diafenthiuron $50 \mathrm{WP}$ and thiamethoxam 25 WG. Whereas, bio pesticides were less effective against all the sucking pests compared to insecticides.

\section{References}

Anonymous, 2014, Package of practices. Univ. Agric. Sci., Dharwad. pp. 181203.

Anonymous, 2015, Impact Evaluation of $\mathrm{Bt}$ cotton in Karnataka. pp. 6

Anonymous, 2016, Agricultural outlook forum: Cotton outlook 2016. p. 1-16. www.usda.gov/oce/forum/2016_speech es/Cotton_Outlook_2016.pdf

Bharpoda, T. M., Patel, N. B., Thumar, R. K., Bhatt, N. A., Ghetiya, I. V., Patel, H. C. and Borad, P. K., 2014, Evaluation of insecticides against sucking insect pests infesting Bt cotton BG-II. The Bioscan., 9(3): 977-980.

Bhavani Sankara Rao, T., Reddy, G. P. V., Murthy, M. M. K. and Deva Prasad, V., 1991, Efficacy of neem products in the control of bhendi pest complex. Indian J. Plant Protec., 19: 49-52.

Gaurkhede, A. S., Bhalkare, S. K., Sadawarte, A. K. and Undirwade, D. B., 2015, Bio efficacy of new chemistry molecules against sucking pests of Bt transgenic cotton. Int. J. Plant Protec., 8 (1): 7-12.

Ghelani, M. K., Kabaria, B. B. and Chhodavadia, S. K., 2014, Field efficacy of various insecticides against major sucking pests of Bt cotton. $J$. Biopest., 7: 27-32.

Ghelani, Y. H., Jhala, R. C. and Vyas, H. N., 2006, Bio efficacy of botanicals and microbial insecticides against cotton aphid, Aphis gossypii (Glover). Adv. Indian Entomol., 2(3): 149-152.

Gomez, J. S. and Gomez, A. A., 1984, Statistical Procedures for Agricultural Research. Second edition. pp. 207-214.

Haegreaves, H., 1948, List of recorded cotton insects of the world. Common Wealth Institute of Entomology, London. pp. 50.

Kadam, D. B., Kadam, D. R., Umate, S. M. and Lekurwale, R. S., 2014, Bio 
efficacy of newer neonicotenoids against sucking insect pests of $\mathrm{Bt}$ cotton. Int. J. Plant Protec., 7 (2): 415 419.

Patil, S. B., Udikeri, S. S., Matti, P. V., Guruprasad, G. S., Hirekurubar, R. B., Shaila, H. M., and Vandal, N. B., 2009, Bio efficacy of new molecule fipronil $5 \%$ SC against sucking pest. Karnataka J. Agric. Sci., 22(5): 1029-1031.

Patil, S. B., Udikeri, S. S., Matti, P. V., Guruprasad, G. S., Hirekurubar, R. B., Shaila, H. M., and Vandal, N. B., 2009, Bio efficacy of new molecule fipronil $5 \%$ SC against sucking pest. Karnataka J. Agric. Sci., 22(5): 1029-1031.

Ravikumar, V., Prasad, N. V. V. S. D. and Madhumathi, T., 2016, Relative toxicity of different insecticides against Thrips tabaci (Lindeman) on cotton. I. J. T. A., 34(5): 1387-1391.
Razaq, M., Anjum, S., Muhammad Aslam, M., Jalal, A., Saleem, M. A. and Khan, M. H. A., 2005, Evaluation of neonicotinoids and conventional insecticides against cotton jassid, Amrasca devastans (Dist.) and cotton whitefly, Bemisia tabaci (Genn.) on cotton. Pak. Entomol., 27(1): 75-78.

Sathyan, T., Murugesan, N., Elanchezhyan, K., Arockia, S. R. J. and Ravi, G., 2016, Efficacy of synthetic insecticides against sucking insect pests in cotton, Gossypium hirsutum L. Int. J. Entomol. Res., 1(1): 16-21.

Uthamasamy S. 1994. Intra and inter plant behavioural dynamics of the cotton bollworm complex. In: Functional Dynamics of Phytophagous Insects (Ed. Ananthakrishnan T. N.), Oxford and IBH Publishers, New Delhi. pp. 115131.

\section{How to cite this article:}

Meghana H., S. B. Jagginavar and Sunitha N. D. 2018. Efficacy of Insecticides and Bio Pesticides against Sucking Insect Pests on Bt Cotton. Int.J.Curr.Microbiol.App.Sci. 7(06): 2872-2883. doi: https://doi.org/10.20546/ijcmas.2018.706.338 